International Conference Mathematical and Computational Biology 2011

International Journal of Modern Physics: Conference Series

Vol. 9 (2012) 560-565

C World Scientific Publishing Company

DOI: $10.1142 / \mathrm{S} 2010194512005661$

\title{
NUMERICAL SOLUTION FOR SOLVING SECOND ORDER ORDINARY DIFFERENTIAL EQUATIONS USING BLOCK METHOD
}

\author{
NUR ZAHIDAH MUKHTAR \\ Institute for Mathematical Research, Universiti Putra Malaysia, \\ 43400 UPM Serdang, Selangor Darul Ehsan, Malaysia \\ bintimukhtar1408@yahoo.com \\ ZANARIAH ABDUL MAJID \\ Mathematics Department, Faculty Science, Universiti Putra Malaysia, \\ 43400 UPM Serdang, Selangor Darul Ehsan, Malaysia \\ zanariah@math.upm.edu.my \\ FUDZIAH ISMAIL \\ Mathematics Department, Faculty Science, Universiti Putra Malaysia, \\ 43400 UPM Serdang, Selangor Darul Ehsan, Malaysia \\ fudziah@math.upm.edu.my \\ MOHAMED SULEIMAN \\ Mathematics Department, Faculty Science, Universiti Putra Malaysia, \\ 43400 UPM Serdang, Selangor Darul Ehsan, Malaysia \\ mohamed@math.upm.edu.my
}

\begin{abstract}
The purpose of this paper is to present a four point direct block one-step method for solving directly the general second order nonstiff initial value problems (IVPs) of ordinary differential equations (ODEs). The mathematical problems in real world can be written in the form of differential equations and arise in the fields of science and engineering such as fluid dynamic, electric circuit, motion of rocket or satellite and other area of application. The proposed method will estimate the approximation solutions at four points simultaneously by using variable step size.

Numerical results are given to show the efficiency of the proposed method.
\end{abstract}

Keywords: Block method; one-step method; ordinary differential equations.

\section{Introduction}

In recent years, there were some problems arises from the fields of science and engineering represented by mathematical models. These mathematical models can be written in the form of differential equations, either as a first order or higher order 
ordinary differential equations (ODEs). This study considers for solving second order nonstiff initial value problems (IVPs) of ODEs of the form

$$
y^{\prime \prime}=f\left(x, y, y^{\prime}\right) \quad y(a)=y_{0}, y^{\prime}(a)=y_{0}^{\prime}, \quad x \in[a, b] .
$$

The approach here is to solve Eq. (1) directly without reducing to first order system ODEs using four point one-step block method. The proposed method has been used to calculate the approximation solution of four points simultaneously in a block. The basic idea of the one-step block method has been studied by Rosser (1967) who introduced a block of new approximation values simultaneously. The approach also been discussed in Worland (1976) and Majid et al. (2003). In Majid et al. (2003), the authors described a two point implicit one-step block method for solving first order ODEs based on integration formula using the closest point in the block.

Equation (1) has already been solved directly by several researchers such as Chakravarti and Worland (1971), Suleiman (1989), Fatunla (1991) and Omar and Suleiman (2005). The system of higher order ODEs can be reduced to a system of first order equation and then solved using first order ODEs. This approach will enlarge the system of first order ODEs and needs more computational work.

The main idea of this research is to extend the work done by Majid et al. (2003) for solving Eq. (1) directly and using variable step size. Numerical results are given to show the efficiency of the proposed method.

\section{Methodology}

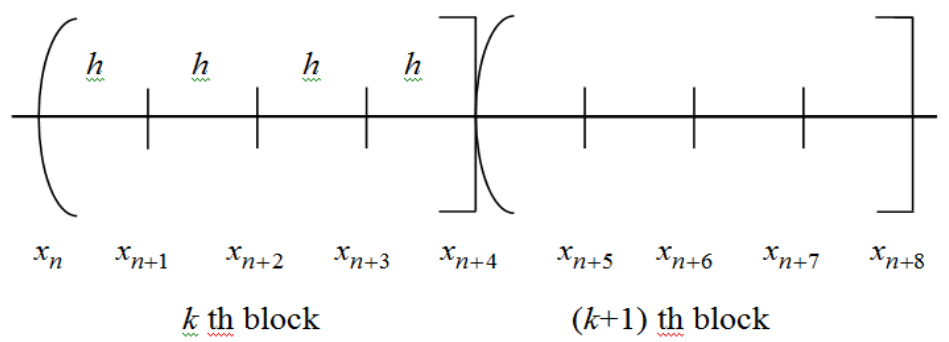

Fig. 1. Four point one-step block method.

In Fig. 1, the approximation solutions of $y_{n+1}, y_{n+2}, y_{n+3}$ and $y_{n+4}$ are simultaneously computed in the block at the points $x_{n+1}, x_{n+2}, x_{n+3}$, and $x_{n+4}$ in $k$ th block. The interval of $[a, b]$ is divided into a series of blocks with each block containing four points with step size, $h$. The predictor formula were obtained from Euler formula meanwhile the corrector formula of the implicit block in Fig. 1 were derived using Lagrange interpolation polynomial. The interpolation points involved are $\left(x_{n}, f_{n}\right), \ldots,\left(x_{n+4}, f_{n+4}\right)$. Then, followed by integrate once and twice on Eqn. (1) over the interval $\left[x_{n}, x_{n+1}\right]$, $\left[x_{n+1}, x_{n+2}\right],\left[x_{n+2}, x_{n+3}\right]$ and $\left[x_{n+3}, x_{n+4}\right]$ respectively using MATHEMATICA. The following corrector formulae will be obtained, 


$$
\begin{aligned}
& y_{n+1}^{\prime}=y_{n}^{\prime}+\frac{h}{720}\left(251 f_{n}+646 f_{n+1}-246 f_{n+2}+106 f_{n+3}-19 f_{n+4}\right) \\
& y_{n+1}=y_{n}+h y_{n}^{\prime}+\frac{h^{2}}{1440}\left(367 f_{n}+540 f_{n+1}-282 f_{n+2}+116 f_{n+3}-21 f_{n+4}\right) \\
& y_{n+2}^{\prime}=y_{n+1}^{\prime}+\frac{h}{720}\left(-19 f_{n}+346 f_{n+1}+456 f_{n+2}-74 f_{n+3}+11 f_{n+4}\right) \\
& y_{n+2}=y_{n+1}+h y_{n+1}^{\prime}+\frac{h^{2}}{1440}\left(-21 f_{n}+472 f_{n+1}+330 f_{n+2}-72 f_{n+3}+11 f_{n+4}\right) \\
& y_{n+3}^{\prime}=y_{n+2}^{\prime}+\frac{h}{720}\left(11 f_{n}-74 f_{n+1}+456 f_{n+2}+346 f_{n+3}-19 f_{n+4}\right) \\
& y_{n+3}=y_{n+2}+h y_{n+2}^{\prime}+\frac{h^{2}}{1440}\left(11 f_{n}-76 f_{n+1}+582 f_{n+2}+220 f_{n+3}-17 f_{n+4}\right) \\
& y_{n+4}^{\prime}=y_{n+3}^{\prime}+\frac{h}{720}\left(-19 f_{n}+106 f_{n+1}-264 f_{n+2}+646 f_{n+3}+251 f_{n+4}\right) \\
& y_{n+4}=y_{n+3}+h y_{n+3}^{\prime}+\frac{h^{2}}{1440}\left(-17 f_{n}+96 f_{n+1}-246 f_{n+2}+752 f_{n+3}+135 f_{n+4}\right)
\end{aligned}
$$

\section{Implementation}

In order to make selection of the next step size, we followed the step size strategy based on Majid et al. (2006) which should it be a successful step, $h$ will remain constant or double. The step size will be restricted to half if the step size failure. The approximation solutions in Eq. (2) will be estimated using the predictor-corrector schemes.

$$
\begin{array}{ll}
y_{n+i}^{\prime}=y_{n+(i-1)}^{\prime}+h f_{n+(i-1)} & \\
y_{n+i}^{\prime}=y_{n+(i-1)}+h y_{n+(i-1)}^{\prime}+\frac{h^{2}}{2 !} f_{n+(i-1)} & i=1,2,3,4
\end{array} .
$$

Define Eq. (3) as the initial approximation and applying the derived method in Eq. (2) as corrector. In order to obtain the corrector iteration is converging, the following convergence test will be used

$$
\left|y_{n+4, r+1}^{c}-y_{n+4, r}^{c}\right|<0.1 \times \text { TOLERANCE. }
$$

where $r$ is the number of iterations. Once the convergence test in Eq. (4) satisfied, then the error for the block will be performed. The maximum error and the average error are defined as below,

$$
\operatorname{MAXE}=\max _{1 \leq i \leq S S}\left(\max _{1 \leq i \leq N}\left(E_{i}\right)\right) \quad \text { and } \mathrm{AVE}=\frac{\sum_{i=1}^{\mathrm{SS}} \sum_{i=1}^{N}\left(E_{i}\right)}{(P)(N)(S S)} .
$$

where $S S$ is the number of successful steps, $\left(E_{i}\right)$ is the error, $P$ is the number of point and $N$ is the number of equations in the system. 


\section{Numerical Results}

The tables below show the numerical results for the two tested problems when solved using the proposed method by $\mathrm{C}$ language code. The following notations in tables will

TOL Tolerance

4POSB Implementation of the direct four point one-step block method proposed in this paper

4PRED Implementation of the four point one-step block method in Radzi, et al. (2011) i.e. reducing to system of first order ODEs

RK45 Solutions obtained by MATLAB built-in solver ode45

TS Total steps taken

MAXE Magnitude of the maximum error

AVE The average error

FCN Total function calls

ABS.ERR Absolute error of the computed solution

Problem 1: The two-body orbit:

$$
\begin{aligned}
& y_{1}^{\prime \prime}=-\frac{y_{1}}{r^{3}}, \quad y_{1}(0)=1, \quad y_{1}^{\prime}(0)=0 \quad r=\sqrt{y_{1}^{2}+y_{2}^{2}} \text {. } \\
& y_{2}^{\prime \prime}=-\frac{y_{2}}{r^{3}}, \quad y_{2}(0)=0, \quad y_{2}^{\prime}(0)=1,
\end{aligned}
$$

Exact solution: $\quad y_{1}(x)=\cos (x), \quad y_{2}(x)=\sin (x)$

Source: Suleiman (1989)

First order systems:

$$
\begin{array}{llll}
y_{1}^{\prime}=y_{3}, & y_{2}^{\prime}=y_{4}, & y_{3}^{\prime}=-\frac{y_{1}}{r^{3}}, & y_{4}^{\prime}=-\frac{y_{2}}{r^{3}} \\
y_{1}(0)=1, & y_{2}(0)=0, & y_{3}(0)=0, & y_{4}(0)=1
\end{array}
$$

Exact solution:

$$
y_{1}(x)=\cos (x), \quad y_{2}(x)=\sin (x), \quad y_{3}(x)=-\sin (x), \quad y_{4}(x)=\cos (x)
$$

Problem 2: Van der Pol oscillator:

$$
y^{\prime \prime}-2 \xi\left(1-y^{2}\right) y^{\prime}+y=0, \quad y(0)=0, \quad y^{\prime}(0)=0.5, \quad[0,40]
$$

Source: Vigo-Aguar and Ramos (2006)

First order systems:

$$
\begin{array}{ll}
y_{1}^{\prime}=y_{2}, & y_{1}(0)=0 \\
y_{2}^{\prime}=2 \xi\left(1-y_{1}{ }^{2}\right) y_{2}-y_{1}, & y_{2}(0)=0.5 \quad \xi=0.025
\end{array}
$$

For Problem 2, the absolute error will be estimate by the following equation as below, ABS. ERR. $\quad y(x)=\left|\bar{y}_{n+4}^{c}-y_{n+4}^{c}\right|, \quad y^{\prime}(x)=\left|\bar{y}_{n+4}^{\prime c}-y_{n+4}^{\prime c}\right|$ 
where $\bar{y}_{n+4}^{c}, \bar{y}_{n+4}^{c}$ denote the corrector formulae at $\mathrm{TOL}=10^{-12}$. Then, we were going to compare the proposed method in this paper with the solutions obtained by the MATLAB built-in solver ode 45 .

Table 1. Comparison between 4POSB and 4PRED methods for solving Problem 1.

\begin{tabular}{cccccc}
\hline \multirow{2}{*}{ TOL } & \multirow{2}{*}{ MTD } & TS & MAXE & AVE & FCN \\
\hline \multirow{2}{*}{$10^{-4}$} & 4PRED & 43 & $6.0784 \mathrm{e}-04$ & $7.1337 \mathrm{e}-05$ & 1017 \\
& 4POSB & 27 & $2.9181 \mathrm{e}-05$ & $9.0450 \mathrm{e}-06$ & 529 \\
\multirow{2}{*}{$10^{-6}$} & 4PRED & 125 & $1.2363 \mathrm{e}-06$ & $2.1477 \mathrm{e}-07$ & 2749 \\
& 4POSB & 57 & $6.4539 \mathrm{e}-06$ & $1.4363 \mathrm{e}-06$ & 909 \\
\multirow{2}{*}{$10^{-8}$} & 4PRED & 207 & $5.9493 \mathrm{e}-09$ & $8.8773 \mathrm{e}-10$ & 5317 \\
& 4POSB & 121 & $3.0989 \mathrm{e}-08$ & $6.6848 \mathrm{e}-09$ & 1937 \\
\multirow{2}{*}{$10^{-10}$} & 4PRED & 637 & $2.1775 \mathrm{e}-11$ & $4.6349 \mathrm{e}-12$ & 14861 \\
& 4POSB & 261 & $1.3901 \mathrm{e}-10$ & $2.9203 \mathrm{e}-11$ & 4177 \\
\hline
\end{tabular}

Table 2. Comparison between 4POSB and RK45 methods for solving Problem 2.

\begin{tabular}{ccccc}
\hline \multirow{2}{*}{ TOL } & MTD & TS & $y(x)$ & $y^{\prime}(x)$ \\
\cline { 3 - 5 } & & & $7.016927 \mathrm{e}-04$ & $4.157618 \mathrm{e}-04$ \\
& & & \multicolumn{2}{c}{ ABS. ERR } \\
\hline \multirow{2}{*}{$10^{-4}$} & RK45 & 285 & $9.610276 \mathrm{e}-04$ & $4.888968 \mathrm{e}-04$ \\
& 4POSB & 43 & $6.567087 \mathrm{e}-06$ & $5.359170 \mathrm{e}-07$ \\
$10^{-6}$ & RK45 & 713 & $4.267339 \mathrm{e}-06$ & $5.667588 \mathrm{e}-06$ \\
& 4POSB & 62 & $5.764219 \mathrm{e}-08$ & $2.270758 \mathrm{e}-08$ \\
$10^{-8}$ & RK45 & 1789 & $4.399870 \mathrm{e}-08$ & $6.230616 \mathrm{e}-08$ \\
& 4 POSB & 122 & $5.331400 \mathrm{e}-10$ & $2.876250 \mathrm{e}-10$ \\
10 & RK45 & 4489 & $4.851652 \mathrm{e}-10$ & $5.869338 \mathrm{e}-10$ \\
\hline
\end{tabular}

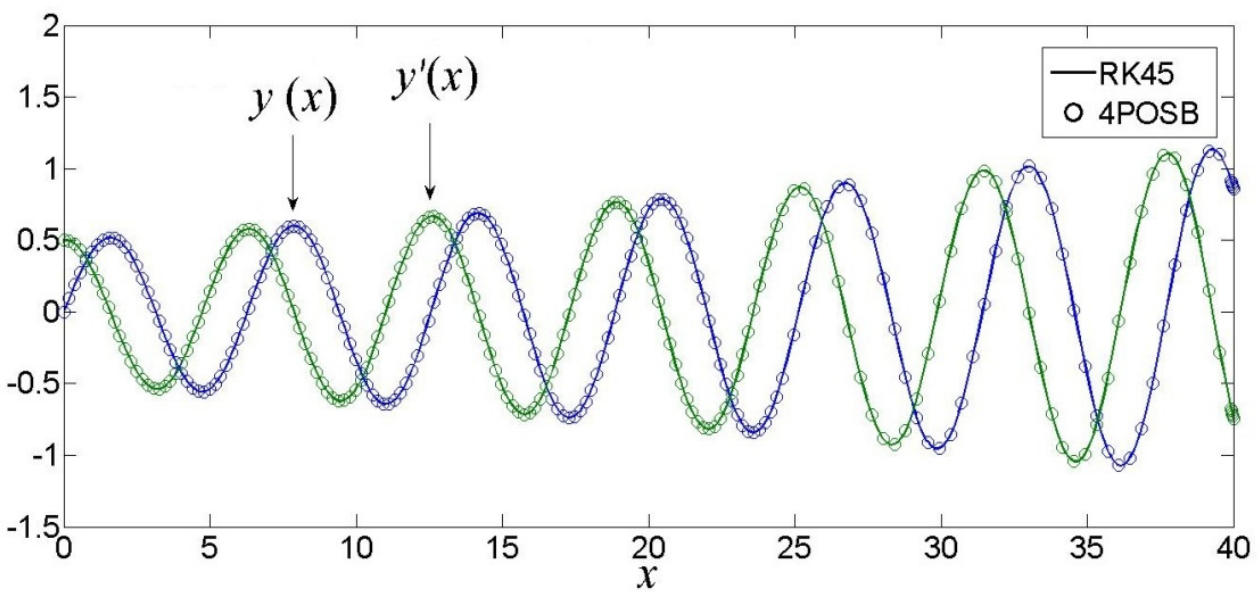

Fig. 2. Solution of Problem 2 by 4 POSB and RK45 at TOL $=10^{-4}$. 


\section{Discussion and Conclusions}

In Table 1 and 2, the numerical results have shown that the proposed method 4POSB reduced the total steps and the total function calls to almost half compared to 4PRED method. These results are expected because 4POSB method solves the problems directly without reducing to system of first order ODEs. It could be observed that the maximum error of 4POSB is comparable and less one decimal place compared to 4PRED in solving Problem 1. For Problem 2, the absolute error of 4POSB is comparable with RK45 (ode45 solver) except less one decimal place at TOL $=10^{-6}$ for $y^{\prime}(x)$ but is still within the given tolerance. Hence, we have shown the 4POBS method is suitable to solve second order ODEs directly and needs less computational work.

\section{Acknowledgments}

The author gratefully acknowledges that this research was partially supported by Universiti Putra Malaysia under the Graduate Research Fellowship (GRF).

\section{References}

1. J. B. Rosser, Runge-Kutta for all seasons, in SIAM Rev. 9, 417-452 (1967).

2. L. F. Shampine and H. F. Watts, Block implicit one-step method, in Math. Comp, 23, 731-740 (1969).

3. P. B. Worland, Parallel methods for the numerical solutions of ordinary differential equations, in IEEE Transactions on Computers, 25, 1045-1048 (1976).

4. Z. A. Majid, M. B. Suleiman, F. Ismail and M. Othman, 2-point implicit block one-step method half Gauss-Seidel for solving first order ordinary differential equations, in Matematika, Jabatan UTM, 19, 91-100 (2003).

5. P. C. Chakravarti and P. B. Worland, A class of self-starting methods for the numerical solution of $y^{\prime \prime}=f(x, y)$, in BIT, 11, 368-383 (1971).

6. M. B. Suleiman, Solving nonstiff higher order ODEs directly by the direct integration method, in Applied Mathematics and Computation, 33, 197-219 (1989).

7. S. O. Fatunla, Block methods for second order ODEs, in International Journal of Computer Mathematics, 41, 55-63 (1991).

8. Z. Omar and M. B. Suleiman, Solving higher order ordinary differential equations using parallel 2-point explicit block method, in Matematika, Jabatan UTM, 21, 15-23 (2005).

9. Z. A. Majid, M. B. Suleiman and Z. Omar, 3-point implicit block method for solving ordinary differential equations, in Bull. Malays. Math. Sci. Soc, 29, 23-31 (2006).

10. J. Vigo-Aguiar and H. Ramos, Variable Stepsize Implementation of Multistep Methods for $y^{\prime \prime}=f\left(x, y, y^{\prime}\right)$, Journal of Computational and Applied Mathematics, 192, 114-131 (2006).

11. H. M. Radzi, Z. A. Majid, F. Ismail and M. B. Suleiman, Four step implicit block method of Runge-Kutta type for solving first order ordinary differential equations. Proceedings of Fourth International Conference on Modeling, Simulation and Applied Optimization 2011, 139-143 (2011). 